Artículo original

(Original paper)

\title{
USO Y VALOR CULTURAL DE LA HERPETOFAUNA EN MÉXICO: UNA REVISIÓN DE LAS ÚLTIMAS DOS DÉCADAS (1997-2017)
}

\section{USE AND CULTURAL VALUE OF THE HERPETOFAUNA IN MEXICO: A REVIEW OF THE LAST TWO DECADES (1997-2017)}

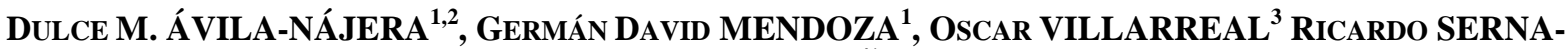 \\ LAGUNES $^{4^{*}}$
${ }^{1}$ Departamento de Producción Agrícola y Animal, Universidad Autónoma Metropolitana, Unidad Xochimilco, Calzada del Hueso 1100, Col. Villa Quietud, D. F. 04960, México
<dul.avna@gmail.com>; <gmendoza@correo.xoc.uam.mx>
${ }^{2}$ Universidad Politécnica de Huatusco. Unidad Académica de Biotecnología y Agroindustrial. Avenida 1 No. 728. Col. Centro. C.P. 94100. Huatusco, Veracruz, México
${ }^{3}$ Facultad de Medicina Veterinaria y Zootecnia, Benemérita Universidad Autónoma de Puebla. Domicilio conocido, El Salado, Tecamachalco, Puebla, México. C. P. 75480.<oscar.villarrealeb@hotmail.com>
${ }^{4}$ Unidad de Manejo y Conservación de Recursos Genéticos. Facultad de Ciencias Biológicas y Agropecuarias, región Orizaba- Córdoba. Universidad Veracruzana. Josefa Ortiz de Domínguez S/N, Col. Centro, C.P. 94945. Peñuela, Municipio de Amatlán de los Reyes, Veracruz, México
*Autor de correspondencia: <rserna@uv.mx>

Recibido: 05/12/2017; aceptado: 08/06/2018; publicado en línea: 12/11/2018 Editor responsable: Gustavo Aguirre

Ávila-Nájera, D. M., Mendoza' J. D., Villarreal O., Serna-Lagunes R. (2018) Uso y valor cultural de la herpetofauna en México: una revisión de las últimas dos décadas (1997-2017). Acta Zoológica Mexicana (nueva serie), 34, 1-15. https://doi.org/10.21829/azm.2018.3412126

RESUMEN. Anfibios y reptiles son grupos de vertebrados que históricamente ha sido utilizado por comunidades rurales. En México, la multiculturalidad del país está estrechamente relacionada con la diversidad biológica con la que coexiste. El uso del Índice de Importancia Cultural (IIC) permite cuantificar el valor de las especies con diversos usos y puede ser de utilidad para enfocar estrategias de conservación, tomando en cuenta el valor cultural de las especies. El objetivo del estudio fue identificar los usos y generar un IIC de la herpetofauna, mediante un meta-análisis bibliométrico de publicaciones sobre el uso de anfibios y reptiles mexicanos. Se registraron 103 reptiles y 32 anfibios que tienen al menos un uso, el cual varió dependiendo del taxón (reptiles o anfibios, respectivamente): alimenticio $(47,10)$, mascota $(20,6)$, comercial $(20,2)$, ornamental $(25,1)$, artesanal $(32,9)$, mágico-religioso (32, 10), medicinal $(57,19)$, caza deportiva $(2,2)$, control de daños $(48,2)$, benéfico $(10,4)$, otros $(3,3)$. Rhinella marina (27.13) y Crocodylus moreletii (15.42) fueron las especies con mayor IIC. De las especies registradas, el $61 \%$ y $31 \%$ de reptiles y anfibios, respectivamente, están en alguna categoría de riesgo. Es necesario establecer estrategias de conservación por medio de manejo y programas educativos que promuevan alternativas de uso sustentable de los recursos, así como la conservación de espacios naturales donde las especies habitan.

Palabras clave: alimenticio, anfibios, índice de importancia cultural, mágico-religioso, medicinal, reptiles. 
Ávila-Nájera, D. M., Mendoza, J. D., Villarreal, O., Serna-Lagunes, R. (2018) Use and cultural value of the herpetofauna in Mexico: a review of the last two decades (1997-2017). Acta Zoológica Mexicana (nueva serie), 34, 1-15. https://doi.org/10.21829/azm.2018.3412126

\begin{abstract}
Amphibians and reptiles are vertebrate groups that historically have been used by rural communities. In Mexico, the multiculturalism of the country is closely related to the biological diversity with which it coexists. The use of the Cultural Importance Index (IIC) allows quantify the value of species with different uses and can be useful to focus conservation strategies. The objective of the study was to identify the use and generate an IIC of the herpetofauna, through a bibliometric meta-analysis of publications on the use of Mexican amphibians and reptiles. There were 103 reptiles and 32 amphibians that have at least one use, which varied depending on the taxon (reptiles or amphibians, respectively): food $(47,10)$, pet $(20,6)$, commercial $(20,2)$, ornamental $(25,1)$, artisan $(32,9)$, magic-religious $(32,10)$, medicinal $(57,19)$, sport hunting $(2,2)$, damage control $(48,2)$, beneficial $(10,4)$. Rhinella marina (27.13) and Crocodylus moreletii (15.42) were the species with the highest IIC. Of the species recorded, 61\% and $31 \%$ of reptiles and amphibians respectively are in some risk category. It is necessary establish conservation strategies through management and education programs that promote sustainable uses of resources, as well as the conservation of natural spaces where these species inhabit.
\end{abstract}

Key words: amphibians, importance cultural index, magic-religious use, medicinal use, nutritional use, reptiles.

\title{
INTRODUCCIÓN
}

Diversos estudios determinan que México es un país que contiene una amplia riqueza cultural (MillánRojas et al., 2016; Rodriguez-Soto et al., 2017), que se relaciona con la diversidad biológica que alberga en su territorio (Azúa \& Estrada, 2015). A nivel mundial, Alves \& van Vliet (2018) han demostrado que el uso de la fauna silvestre es variante en el tiempo y el espacio, ya que intrínsecamente está relacionada con los hábitos culturales, formas de vida y las prácticas de manejo del ecosistema, mismos que cambian dependiendo el grupo social, su historia, posición geográfica (Toledo \& Barrera, 2008) y desarrollo socioeconómico (Vázquez-Dávila, 1999). Esta diversidad de usos se puede registrar y estudiar mediante técnicas de cosmovisión, agrupándolos en tres vertientes de conocimiento: cosmos (relación sociedadnaturaleza), corpus (conocimiento cognitivo de los recursos naturales) y práxis (apropiación de la diversidad biológica [Cabrera et al., 2001]).

Dentro de los grupos de vertebrados que tienen una relación intrínseca con la sociedad rural, desde el enfoque etnozoológico, está la herpetofauna. Este grupo, en diversas culturas, forma parte importante de su consmovisión (González, 2001). Para México, se han estimado 864 especies de reptiles y 376 especies de anfibios (Flores-Villela \& García-Vázquez, 2013; Parra-Olea et al., 2014), posicionando al país en el segundo y quinto lugar, respectivamente, a nivel mundial. Sin embargo, AmphibiaWeb (2017) tiene un registro de 838 especies, mientras que Frost (2017) señala 403 anfibios; y por otro lado, Uetz et al. (2018) enlistan 936 especies de reptiles. Esta riqueza de anfibios y reptiles coexiste en el entorno con al menos 67 lenguas nativas que radican en el territorio mexicano. Por tanto, no es circunstancial que aproximadamente 517 especies de herpetozoos estén arraigadas en esquemas simbólicos (Casas-Andreu, 2008) espirituales y sociales de pueblos indígenas y no indígenas (Macip-Ríos \& Casas-Andreu, 2008).

A pesar de que la fauna silvestre constituye un elemento natural significativo en el desarrollo histórico y evolutivo de la cultura humana (Santos-Fita et al., 2012), particularmente los anfibios y reptiles han formado parte importante de la cultura e identidad mexicana, como es en la expresión humana, usos prácticos y cosmovisión de las culturas prehispánicas, esto hace que sea preponderante registrar la 
información de estos taxa, documentar información que permita entender el uso y valor de este recurso natural que la cultura mexicana actual les otorga (Aguilar \& Luría, 2016). Es poco el registro que se tiene de un grupo tan diverso como son los anfibios y reptiles. Es decir, se desconoce el valor cuantitativo de este grupo taxonómico, que indique desde una perspectiva cultural, el valor que adquiere cada especie. En este sentido, el objetivo fue documentar los usos y el valor cultural reciente de la herpetofauna de México, mediante la recopilación de trabajos publicados en las últimas dos décadas (1997-2017) y generar un Índice de Importancia Cultural (IIC) que cuantifique la intensidad de los usos y menciones de cada una de las especies registradas.

\section{MATERIALES y MÉTODOS}

Se realizó un meta-análisis bibliométrico (Albuquerque \& Medeiros, 2012) basado en la revisión sistemática de literatura científica publicada en las dos últimas décadas sobre estudios etnobiológicos de la herpetofauna en México (1997-2017); como criterio de elección de literatura científica, esta debió abordar la temática sobre el uso y valor otorgados a anfibios y reptiles de México. Este se aplicó en la base de datos de la biblioteca virtual del Consorcio Nacional de Recursos de Información Científica y Tecnológica (CONRICyT), además de fuentes publicadas en internet, bases de datos de universidades de México y el extranjero y solicitudes a investigadores. La información recopilada sobre el uso de anfibios y reptiles se capturó en el software Excel ${ }^{\circledR}$ de Microsoft.

Además del meta-análisis, se realizó una consulta a expertos, para agrupar los diferentes usos mencionados en la literatura para clasificarlos; se define al uso como la satisfacción de necesidades concretas de la sociedad (García \& Monroy, 2010). Estas categorías de uso fueron: 1) alimenticio, 2) medicinal, 3) mágico-religioso 4) artesanal 5) comercial, 7) mascota, 8) caza deportiva (Monroy \& García, 2013), 9) control de daños (animales que perjudican los cultivos y que se encuentran principalmente en áreas agrícolas o por alimentarse de especies domésticas, pero en general representan pérdidas económicas o daños a la salud [Morales-Mavil \& Villa-Cañedo, 1998]) y 10) benéficas (aquellas especies que se reconocen por su función ecológica, por ejemplo: el mantenimiento y control de poblaciones nocivas [Cossio, 2007]). Esta información se analizó con tablas de frecuencias de mención para estimar el IIC.

Con la información recopilada, se generó el Índice de Importancia Cultural $\left(I I C_{\mathrm{z}}\right)$ propuesto por Turner (1988), y que ha sido modificado para su aplicación en fauna silvestre (Ávila-Nájera et al., 2011). Con la ecuación: $I I C_{z}=\sum\left(I u_{z}+F m_{z}+V u_{t z}\right)$ se obtuvo el valor cuantitativo de uso para la herpetofauna analizada; donde $I I C_{Z}$ : Índice de Importancia Cultural de una especie determinada; $I u_{z}$ : Intensidad de uso; $F m_{z}$ : Frecuencia de mención; $V u_{t z}$ : Valor de uso total para la especie $z$. Estas variables se calcularon de la siguiente forma:

$I u_{z}$ : número de usos de la especie $z$ de todos los informantes dividido entre el número total de usos de todas las especies y de todos los informantes, multiplicado por 100.

$\mathrm{Fm}_{\mathrm{z}}$ : número de menciones de la especie $\mathrm{z}$ de todos los usos y de todos los informantes dividido entre el número total de menciones de todas las especies para todos los usos y de todos los informantes, multiplicado por 100 .

$V u_{x}$ : número de menciones de la especie $z$ para un uso de todos los informantes dividido entre el número total de menciones de todas las especies para un uso $x$ y de todos los informantes, multiplicado por 100 .

$$
V u_{t z}=\sum\left(V u_{x}+V u_{y}+V u_{z}+\cdots+V u_{n}\right)
$$

El IIC se calculó por separado, es decir, uno con la información de especies de reptiles y uno con la información recopilada para anfibios. La actualización taxonómica de los nombres científicos de 
anfibios y reptiles se realizó con base en las referencias de Frost (2018, Amphibian Species of the World: an Online Reference) y Uetz et al. (2018, The Reptile Database).

El estatus de conservación de las especies se reportó de acuerdo a SEMARNAT (2010). Es importante precisar que Heloderma horridum, Boa constrictor, Crotalus durisssus y Rhinella marina han tenido una revisión taxonómica conforme a la literatura señalada, aunque la NOM-059-SEMARNAT2010 todavía no refleja los cambios taxonómicos. Para el caso de Chelydra serpentina y Chelydra rossignoni reportada en la literatura, los valores de ambas especies fueron usados para calcular el IIC de C. rossignoni, ya que de acuerdo con Turtle Taxonomy Working Group (2017), esta es la especie válida con distribución en México y su estatus de conservación en la Norma corresponde a C. serpentina (SEMARNAT, 2010).

Cuadro 1. Publicaciones sobre el uso de la herpetofauna en México de 1997 a 2017 empleadas para el cálculo del Î́ndice de Importancia Cultural (IIC).

\begin{tabular}{ll}
\hline \multicolumn{1}{c}{ Estado } & \multicolumn{1}{c}{ Publicaciones } \\
\hline Aguascalientes & Vázquez-Días \& Quintero-Días, 2012; Amador-Alcalá \& De la Riva, 2016 \\
Campeche & Méndez, 2005; León-Martínez, 2006; Méndez-Cabrera \& Montiel, 2007; León \& Montiel, \\
& 2009; Santos-Fita et al., 2012; Ramírez-Mella et al., 2016. \\
Ciudad de & Aranda et al., 1999; Álvarez \& Pacheco, 2010; Gómez-Álvarez \& Pacheco, 2010; Balderas et \\
México & al., 2014; Navarijo-Ornelas, 2014. \\
Chiapas & March, 1996; Cupul-Magaña 2003; Naranjo et al., 2004; Enríquez-Vázquez et al., 2006; \\
& Nucadami, 2006; Naranjo \& Cuarón, 2010; González-Bocanegra et al., 2011; Serrano-González \\
& et al., 2011; Barrasa, 2012; Naranjo, 2013; Tejeda-Cruz et al., 2014. \\
Durango & Jacobo-Salcedo, 2010. \\
Edo. de México & Aranda et al., 1999; Monroy-Vilchis et al., 2008; Guerrero \& Retana, 2012; Navarijo-Ornelas, \\
& 2014. \\
Guanajuato & Naranjo \& Cuarón, 2010; Uriarte-Garzón, 2012; Navarijo-Ornelas, 2014; Leyte-Manrique et \\
& al., 2016. \\
Guerrero & Naranjo \& Cuarón, 2010. \\
Hidalgo & Penguilly et al., 2010. \\
Jalisco & Martínez-Ibarra \& Arellano-Montoya, 2010; Naranjo \& Cuarón, 2010; Navarijo-Ornelas, 2014. \\
México (país) & Casas-Andreu, 2000; Casas-Andreu, 2008; Corona, 2008; Alonso-Castro, 2014. \\
Michoacán & Sánchez, 2006; Naranjo y Cuarón, 2010. \\
Morelos & Monroy \& García, 2013; Navarijo-Ornelas, 2014; Juárez-Mondragón et al., 2015; Reyna-Rojas \\
& et al., 2015. \\
Oaxaca & Naranjo \& Cuarón, 2010; Lira-Torres et al., 2014. \\
Puebla & Cossio, 2007; Navarijo-Ornelas, 2014. \\
Querétaro & Navarijo-Ornelas, 2014. \\
Quintana Roo & Santos-Fita et al., 2012. \\
San Luis Potosí & Navarijo-Ornelas, 2014. \\
Sinaloa & Cortés-Gregorio et al., 2013; Pascual-Ramos et al., 2014. \\
Tabasco & Centeno-Pérez \& Arriaga-Weiss, 2010; Hernández-López et al., 2013. \\
Veracruz & Morales-Mavíl \& Villa-Cañedo, 1998; Morales-Mavíl \& Suárez-Castillo, 2010; Gómez-Landa, \\
Yucatán & 2015. \\
\hline
\end{tabular}

\section{RESULTADOS}

Del total de trabajos recopilados en las últimas dos décadas, el 70\% hacen mención al uso de anfibios y/o reptiles (Cuadro 1). Los estudios analizados usaron entrevistas como instrumento de recopilación de información y se llevaron a cabo en más de 250 comunidades rurales, pertenecientes a 21 estados de la 
República Mexicana (Aguascalientes, Campeche, Ciudad de México, Chiapas, Durango, Estado de México, Guanajuato, Guerrero, Hidalgo, Jalisco, Michoacán, Morelos, Oaxaca, Puebla, Querétaro, Quintana Roo, San Luis Potosí, Sinaloa, Tabasco, Veracruz y Yucatán).

La herpetofauna reportada estuvo representada de la siguiente manera: 28 familias, 58 géneros y 103 especies para reptiles y en 11 familias, 18 géneros y 32 especies para anfibios. Se encontraron diferentes intensidades de uso, frecuencias de mención y valores de uso (Cuadro 2). El número de especies de reptiles y anfibios por uso respectivamente fue: alimenticio (47, 10), mascota (20,6), comercial (20, 2), ornamental $(25,1)$, artesanal $(32,9)$, mágico-religioso $(32,10)$, medicinal $(57,19)$, caza deportiva $(2,2)$, control de daños $(48,2)$, benéfico $(10,4)$. Los dos usos más reconocidos por los encuestados fueron el alimenticio y medicinal para ambos grupos (Fig. 1).

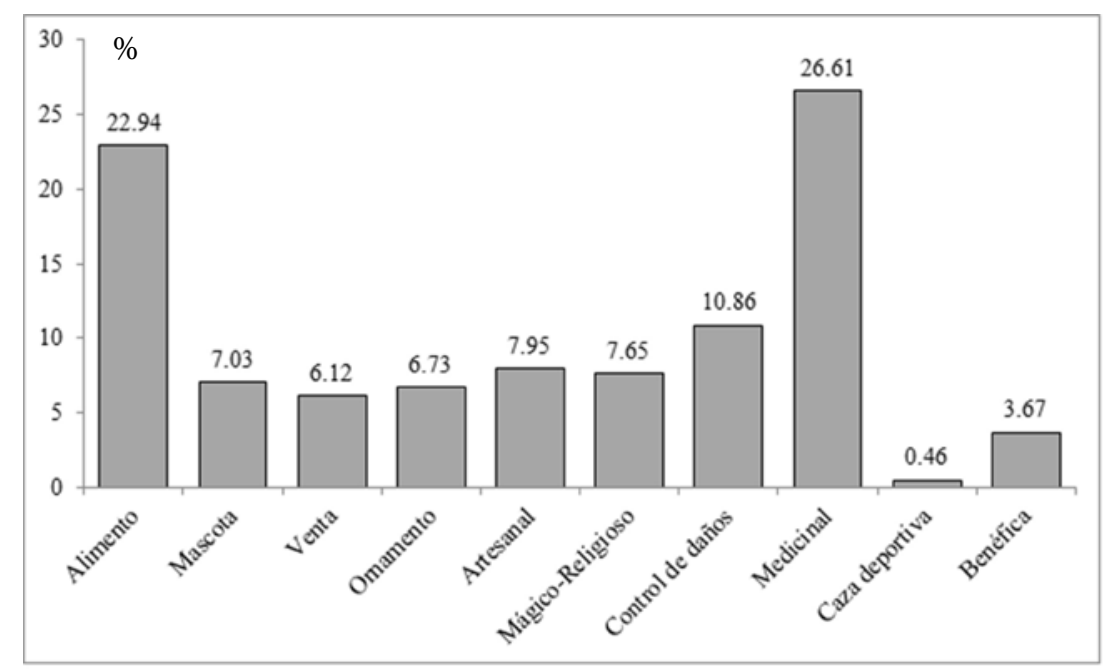

Figura 1. Porcentaje de menciones por uso de la herpetofauna en México, reportada en la literatura en las últimas dos décadas (1997-2017).

Los anfibios con mayor ICC fueron Rhinella marina $\left(I I C_{z}=27.13\right)$, Lithobates berlandieri $\left(I I C_{z}=22.78\right)$ y Lithobates neovolcanicus $\left(I I C_{z}=19.27\right)$, estas dos últimas se encuentran bajo protección ya que se consideran amenazadas. Por su parte, los reptiles con un mayor IIC fueron: Crocodylus moreletii $\left(I I C_{z}=15.42\right)$, Ctenosaura similis $\left(I I C_{z}=14.43\right)$ y Ctenosaura pectinata $\left(I I C_{z}=14.31\right)$, la primera se encuentra protegida y las dos últimas en grado de amenaza (Cuadro 2).

De acuerdo con la NOM-059-SEMARNAT-2010, en este estudio, 64 especies de reptiles y 10 especies de anfibios se consideran en alguna categoría de riesgo. La categoría de riesgo estuvo representada para reptiles en 36 especies bajo la categoría de protección (Pr), 21 especies amenazadas (A), y siete en peligro (P). Para anfibios, siete especies estuvieron en la categoría de protección (Pr), dos especies amenazadas (A) y una en peligro (P) (Cuadro 2).

\section{DISCUSIÓN}

Realizar una recopilación de los trabajos etnozoológicos publicados en México en las dos últimas décadas permitió reconocer el uso de la herpetofauna y su valor cultural (significado o conocimiento del grupo social que maneja el recurso; Colín \& Monroy, 2004). En este trabajo se identificó que en México, 103 especies de reptiles y 32 especies de anfibios tienen al menos un uso y una mención, lo que representa el 
11\% de especies de reptiles y el $8 \%$ de las especies de anfibios descritas para México. Entre los usos identificados destaca el uso mágico-religioso, mismo que está estrechamente relacionado con el uso medicinal, seguido del alimenticio. La Familia Viperidae, representada principalmente por el género Crotalus, destacó por el número de menciones en el uso medicinal y el mágico-religioso. Tradicionalmente, estos vipéridos son usados para curar cáncer, diabetes, acné y manchas en la piel (Gómez-Álvarez \& Pacheco, 2010).

Cuadro 2. Índice de Importancia Cultural (IIC) y número de usos de anfibios y reptiles reportados en publicaciones en las dos últimas décadas en México (1997-2017). Lu: Intensidad de Uso, Fm: Frecuencia de mención, VuT: Valor de uso total para cada especie. Categoría de Riesgo: Pr: sujetas a protección especial, P: en peligro de extinción, A: Amenazada (NOM-059-SEMARNAT-2010). Los valores resaltados en negritas indican el mayor IIC.

\begin{tabular}{|c|c|c|c|c|c|c|c|}
\hline & Categoría & $\begin{array}{c}\text { No. de } \\
\text { usos }\end{array}$ & $\begin{array}{c}\text { Total de } \\
\text { menciones }\end{array}$ & Lu & Fm & VuT & ICC \\
\hline \multicolumn{8}{|c|}{ ORDEN CROCODYLIA Owen, 1842} \\
\hline \multicolumn{8}{|c|}{ FAMILIA ALLIGATORIDAE Gray, 1844} \\
\hline Caiman crocodilus & $\operatorname{Pr}$ & 7 & 14 & 2.36 & 2.43 & 2.31 & 7.09 \\
\hline \multicolumn{8}{|c|}{ FAMILIA CROCODYLIDAE Gray, 1825} \\
\hline Crocodylus acutus & $\operatorname{Pr}$ & 8 & 22 & 2.69 & 3.81 & 4.46 & 10.96 \\
\hline Crocodylus moreletii & $\operatorname{Pr}$ & 9 & 32 & 3.03 & 5.55 & 6.84 & 15.42 \\
\hline \multicolumn{8}{|c|}{ ORDEN SQUAMATA Oppel, 1811} \\
\hline \multicolumn{8}{|c|}{ FAMILIA ANGUIDAE Cope, 1864} \\
\hline Abronia lythrochila & A & 1 & 2 & 0.34 & 0.35 & 0.14 & 0.82 \\
\hline Abronia taeniata & $\operatorname{Pr}$ & 2 & 2 & 0.67 & 0.35 & 0.39 & 1.41 \\
\hline Barisia ciliaris & & 2 & 3 & 0.67 & 0.52 & 0.54 & 1.73 \\
\hline Barisia imbricata & $\operatorname{Pr}$ & 3 & 4 & 1.01 & 0.69 & 0.91 & 2.61 \\
\hline Gerrhonotus liocephalus & $\operatorname{Pr}$ & 1 & 1 & 0.34 & 0.17 & 0.14 & 0.65 \\
\hline Mesaspis moreleti & $\operatorname{Pr}$ & 1 & 2 & 0.34 & 0.35 & 0.14 & 0.82 \\
\hline \multicolumn{8}{|l|}{ FAMILIA BOIDAE Gray, 1825} \\
\hline Boa constrictor & A & 8 & 31 & 2.69 & 5.37 & 5.47 & 13.54 \\
\hline \multicolumn{8}{|c|}{ FAMILIA COLUBRIDAE Oppel, 1811} \\
\hline Conopsis biserialis & A & 1 & 1 & 0.34 & 0.17 & 0.56 & 1.07 \\
\hline Conopsis lineata & & 2 & 4 & 0.67 & 0.69 & 1.11 & 2.48 \\
\hline Conopsis nasus & & 2 & 2 & 0.67 & 0.35 & 0.41 & 1.43 \\
\hline Diadophis punctatus & & 1 & 1 & 0.34 & 0.17 & 0.14 & 0.65 \\
\hline Drymarchon corais & & 2 & 3 & 0.67 & 0.52 & 0.54 & 1.73 \\
\hline Drymarchon melanurus & & 5 & 5 & 1.68 & 0.87 & 0.83 & 3.38 \\
\hline Drymobius margaritiferus & & 2 & 4 & 0.67 & 0.69 & 0.41 & 1.78 \\
\hline Geophis dugesii & & 1 & 1 & 0.34 & 0.17 & 0.14 & 0.65 \\
\hline Geophis hoffmanni & & 1 & 2 & 0.34 & 0.35 & 0.15 & 0.83 \\
\hline Heterodon nasicus & $\operatorname{Pr}$ & 1 & 1 & 0.34 & 0.17 & 0.14 & 0.65 \\
\hline Lampropeltis mexicana & A & 1 & 1 & 0.34 & 0.17 & 0.26 & 0.77 \\
\hline Lampropeltis triangulum & A & 3 & 4 & 1.01 & 0.69 & 0.49 & 2.19 \\
\hline Masticophis bilineatus & & 1 & 2 & 0.34 & 0.35 & 0.29 & 0.97 \\
\hline Masticophis flagellum & A & 1 & 1 & 0.34 & 0.17 & 0.14 & 0.65 \\
\hline Masticophis mentovarius & A & 2 & 5 & 0.67 & 0.87 & 1.24 & 2.78 \\
\hline Masticophis taeniatus & & 1 & 1 & 0.34 & 0.17 & 0.14 & 0.65 \\
\hline Oxybelis aeneus & & 2 & 3 & 0.67 & 0.52 & 0.54 & 1.73 \\
\hline Pituaphis deppei & A & 4 & 4 & 1.35 & 0.69 & 0.61 & 2.65 \\
\hline Pantherophis obsoletus & & 1 & 2 & 0.34 & 0.35 & 0.15 & 0.83 \\
\hline Senticolis triaspis & & 1 & 1 & 0.34 & 0.17 & 0.14 & 0.65 \\
\hline Spilotes pullatus & & 5 & 6 & 1.68 & 1.04 & 0.89 & 3.61 \\
\hline Storeria storerioides & & 1 & 1 & 0.34 & 0.17 & 0.14 & 0.65 \\
\hline
\end{tabular}




\begin{tabular}{|c|c|c|c|c|c|c|c|}
\hline & Categoría & $\begin{array}{c}\text { No. de } \\
\text { usos }\end{array}$ & $\begin{array}{c}\text { Total de } \\
\text { menciones }\end{array}$ & Lu & Fm & VuT & ICC \\
\hline Tantilla bocourti & & 1 & 1 & 0.34 & 0.17 & 0.14 & 0.65 \\
\hline Thamnophis eques & A & 1 & 2 & 0.34 & 0.35 & 0.53 & 1.21 \\
\hline Thamnophis scalaris & A & 2 & 2 & 0.67 & 0.35 & 0.38 & 1.40 \\
\hline Thamnophis sirtalis & $\operatorname{Pr}$ & 1 & 1 & 0.34 & 0.17 & 0.23 & 0.74 \\
\hline Trimorphodon tau & & 1 & 1 & 0.34 & 0.17 & 0.14 & 0.65 \\
\hline \multicolumn{8}{|c|}{ FAMILIA CORYTOPHANIDAE Fitzinger, 1843} \\
\hline Basiliscus vittatus & & 1 & 1 & 0.34 & 0.17 & 0.07 & 0.58 \\
\hline \multicolumn{8}{|c|}{ FAMILIA ELAPIDAE Boie, 1827} \\
\hline Micrurus browni & $\operatorname{Pr}$ & 1 & 1 & 0.34 & 0.17 & 0.20 & 0.71 \\
\hline Micrurus diastema & $\operatorname{Pr}$ & 1 & 1 & 0.34 & 0.17 & 0.14 & 0.65 \\
\hline Micrurus distans & $\operatorname{Pr}$ & 1 & 1 & 0.34 & 0.17 & 0.14 & 0.65 \\
\hline Micrurus euryxanthus & & 2 & 4 & 0.67 & 0.69 & 0.56 & 1.92 \\
\hline Micrurus tener & & 1 & 1 & 0.34 & 0.17 & 0.14 & 0.65 \\
\hline \multicolumn{8}{|c|}{ FAMILIA GEKKONIDAE Stejneger, 1907} \\
\hline Hemidactylus frenatus & & 2 & 3 & 0.67 & 0.52 & 0.64 & 1.84 \\
\hline Phyllodactylus lanei & & 1 & 1 & 0.34 & 0.17 & 0.07 & 0.58 \\
\hline \multicolumn{8}{|c|}{ FAMILIA HELODERMATIDAE Gray, 1837} \\
\hline Heloderma horridum & A & 5 & 6 & 1.68 & 1.04 & 1.18 & 3.90 \\
\hline Heloderma suspectum & A & 1 & 1 & 0.34 & 0.17 & 0.25 & 0.76 \\
\hline \multicolumn{8}{|c|}{ FAMILIA IGUANIDAE Oppel, 1811} \\
\hline Ctenosaura acanthura & $\operatorname{Pr}$ & 3 & 3 & 1.01 & 0.52 & 0.38 & 1.91 \\
\hline Ctenosaura pectinata & A & 5 & 26 & 1.68 & 4.51 & 8.12 & 14.31 \\
\hline Ctenosaura similis & A & 8 & 21 & 2.69 & 3.64 & 8.10 & 14.43 \\
\hline Iguana iguana & $\operatorname{Pr}$ & 6 & 34 & 2.02 & 5.89 & 5.07 & 12.99 \\
\hline \multicolumn{8}{|c|}{ FAMILIA PHRYNOSOMATIDAE Blainville, 1835} \\
\hline Phrynosoma orbiculare & A & 3 & 7 & 1.01 & 1.21 & 1.00 & 3.22 \\
\hline Phrynosoma taurus & A & 2 & 2 & 0.67 & 0.35 & 0.28 & 1.30 \\
\hline Sceloporus grammicus & $\operatorname{Pr}$ & 4 & 7 & 1.35 & 1.21 & 2.14 & 4.70 \\
\hline Sceloporus serrifer & A & 1 & 4 & 0.34 & 0.69 & 0.28 & 1.31 \\
\hline Sceloporus spinosus & & 5 & 6 & 1.68 & 1.04 & 1.34 & 4.06 \\
\hline Sceloporus taeniocnemis & & 1 & 1 & 0.34 & 0.17 & 0.07 & 0.58 \\
\hline Sceloporus torcuatus & & 5 & 9 & 1.68 & 1.56 & 1.62 & 4.87 \\
\hline Seceloporus variabilis & & 1 & 1 & 0.34 & 0.17 & 0.07 & 0.58 \\
\hline \multicolumn{8}{|c|}{ FAMILIA POLYCHRIDAE Frost \& Etheridge, 1989} \\
\hline \multirow{2}{*}{\multicolumn{8}{|c|}{ FAMIIA SCINCIDAE Gray, 1825}} \\
\hline & & & & & & & \\
\hline Eumeces obsoletus & & 1 & 2 & 0.34 & 0.35 & 0.15 & 0.83 \\
\hline Plestiodon lynxe & $\operatorname{Pr}$ & 2 & 2 & 0.67 & 0.35 & 0.39 & 1.41 \\
\hline \multicolumn{8}{|c|}{ FAMILIA TEIIDAE Wiegmann, 1834} \\
\hline Aspidoscelis gularis & & 2 & 3 & 0.67 & 0.52 & 0.54 & 1.73 \\
\hline Holcosus undulatus & & 1 & 1 & 0.34 & 0.17 & 0.25 & 0.76 \\
\hline \multicolumn{8}{|c|}{ FAMILIA TYPHLOPIDAE Daudin, 1803} \\
\hline \multirow{2}{*}{\multicolumn{8}{|c|}{ FAMILIA VIPERIDAE Oppel, 1811}} \\
\hline & & & & & & & \\
\hline Agkistrodon bilineatus & $\operatorname{Pr}$ & 2 & 6 & 0.67 & 1.04 & 0.99 & 2.70 \\
\hline Atropoides nummifer & A & 2 & 3 & 0.67 & 0.52 & 0.34 & 1.54 \\
\hline Bothrops asper & & 6 & 13 & 2.02 & 2.25 & 1.93 & 6.20 \\
\hline Cerrophidion tzotzilorum & $\operatorname{Pr}$ & 2 & 4 & 0.67 & 0.69 & 0.41 & 1.78 \\
\hline Crotalus aquilus & $\operatorname{Pr}$ & 4 & 7 & 1.35 & 1.21 & 0.90 & 3.46 \\
\hline Crotalus atrox & $\operatorname{Pr}$ & 2 & 5 & 0.67 & 0.87 & 0.53 & 2.07 \\
\hline Crotalus basiliscus & $\operatorname{Pr}$ & 2 & 2 & 0.67 & 0.35 & 0.30 & 1.32 \\
\hline Crotalus culmitatus & & 7 & 17 & 2.36 & 2.95 & 2.64 & 7.94 \\
\hline Crotalus durissus & $\operatorname{Pr}$ & 6 & 7 & 2.02 & 1.21 & 1.10 & 4.33 \\
\hline Crotalus lepidus & $\operatorname{Pr}$ & 3 & 3 & 1.01 & 0.52 & 0.38 & 1.91 \\
\hline
\end{tabular}




\begin{tabular}{|c|c|c|c|c|c|c|c|}
\hline & Categoría & $\begin{array}{c}\text { No. de } \\
\text { usos }\end{array}$ & $\begin{array}{c}\text { Total de } \\
\text { menciones }\end{array}$ & Lu & Fm & VuT & ICC \\
\hline Crotalus molossus & $\operatorname{Pr}$ & 7 & 16 & 2.36 & 2.77 & 2.19 & 7.32 \\
\hline Crotlus polistyctus & $\operatorname{Pr}$ & 3 & 4 & 1.01 & 0.69 & 0.45 & 2.15 \\
\hline Crotalus pricei & $\operatorname{Pr}$ & 3 & 3 & 1.01 & 0.52 & 0.38 & 1.91 \\
\hline Crotalus ravus & A & 7 & 10 & 2.36 & 1.73 & 1.79 & 5.88 \\
\hline Crotalus scutulatus & $\operatorname{Pr}$ & 4 & 5 & 1.35 & 0.87 & 0.70 & 2.92 \\
\hline Crotalus simus & & 1 & 1 & 0.34 & 0.17 & 0.07 & 0.58 \\
\hline Crotalus transversus & $\operatorname{Pr}$ & 2 & 2 & 0.67 & 0.35 & 0.14 & 1.16 \\
\hline Crotalus triseriatus & & 4 & 6 & 1.35 & 1.04 & 0.68 & 3.07 \\
\hline Crotalus tzabcan & & 5 & 11 & 1.68 & 1.91 & 1.15 & 4.74 \\
\hline \multicolumn{8}{|c|}{ ORDEN TESTUDINES Linnaeus, 1758} \\
\hline \multicolumn{8}{|c|}{ FAMILIA BATAGURIDAE Gray, 1869} \\
\hline Rhinoclemmys areolata & A & 4 & 6 & 1.35 & 1.04 & 1.00 & 3.39 \\
\hline \multicolumn{8}{|c|}{ FAMILIA CHELONIIDAE Oppel, 1811} \\
\hline Caretta caretta & $\mathrm{P}$ & 2 & 2 & 0.67 & 0.35 & 0.14 & 1.16 \\
\hline Chelonia mydas & $\mathrm{P}$ & 3 & 5 & 1.01 & 0.87 & 0.68 & 2.55 \\
\hline Lepidochelys olivacea & $\mathrm{P}$ & 3 & 5 & 1.01 & 0.87 & 0.68 & 2.55 \\
\hline \multicolumn{8}{|c|}{ FAMILIA CHELYDRIDAE Swainson, 1839} \\
\hline Chelydridae rossignoni & $\operatorname{Pr}$ & 4 & 9 & 1.67 & 1.35 & 0.94 & 3.45 \\
\hline \multicolumn{8}{|c|}{ FAMILIA DERMATEMYDAE Gray, 1870} \\
\hline Dermatemys mawii & $\mathrm{P}$ & 4 & 11 & 1.35 & 1.91 & 1.38 & 4.64 \\
\hline \multicolumn{8}{|c|}{ FAMILIA DERMOCHELYDAE Fitzinger, 1843} \\
\hline Dermochelys coriacea & $\mathrm{P}$ & 2 & 2 & 0.67 & 0.35 & 0.28 & 1.30 \\
\hline \multicolumn{8}{|c|}{ FAMILIA EMYDIDAE Rafinesque, 1884} \\
\hline Terrapene carolina & $\operatorname{Pr}$ & 3 & 4 & 1.01 & 0.69 & 0.48 & 2.18 \\
\hline Trachemys nebulosa & & 1 & 2 & 0.34 & 0.35 & 0.15 & 0.83 \\
\hline Trachemys scripta & $\operatorname{Pr}$ & 6 & 19 & 2.02 & 3.29 & 3.07 & 8.38 \\
\hline Trachemys venusta & & 3 & 3 & 1.01 & 0.52 & 0.40 & 1.93 \\
\hline \multicolumn{8}{|c|}{ FAMILIA KINOSTERNIDAE Agassiz, 1857} \\
\hline Claudius angustatus & $\mathrm{P}$ & 3 & 4 & 1.01 & 0.69 & 0.47 & 2.17 \\
\hline Kinosternon acutum & $\operatorname{Pr}$ & 4 & 10 & 1.35 & 1.73 & 1.04 & 4.12 \\
\hline Kinosternon hirtipes & $\operatorname{Pr}$ & 1 & 1 & 0.34 & 0.17 & 0.07 & 0.58 \\
\hline Kinosternon integrum & $\operatorname{Pr}$ & 7 & 11 & 2.36 & 1.91 & 1.64 & 5.90 \\
\hline Kinosternon leucostomum & $\operatorname{Pr}$ & 5 & 10 & 1.68 & 1.73 & 1.20 & 4.61 \\
\hline Staurotypus salvini & $\operatorname{Pr}$ & 2 & 2 & 0.67 & 0.35 & 0.28 & 1.30 \\
\hline Staurotypus triporcatus & A & 5 & 14 & 1.68 & 2.43 & 2.02 & 6.13 \\
\hline \multicolumn{8}{|c|}{ FAMILIA TESTUDINIDAE Batsch, 1788} \\
\hline Gopherus flavomarginatus & $\mathrm{P}$ & 1 & 2 & 0.34 & 0.35 & 0.14 & 0.82 \\
\hline \multicolumn{8}{|c|}{ ORDEN ANURA Fischer von Waldheim, 1813} \\
\hline \multicolumn{8}{|c|}{ FAMILIA AMBYSTOMATIDAE Gray, 1850} \\
\hline Ambystoma mexicanum & $\mathrm{P}$ & 2 & 2 & 3.39 & 2.20 & 4.63 & 10.22 \\
\hline Ambystoma tigrinum & & 1 & 1 & 1.69 & 1.10 & 4.20 & 6.99 \\
\hline \multicolumn{8}{|c|}{ FAMILIA BUFONIDAE Gray, 1825} \\
\hline Anaxyrus compactilis & & 1 & 1 & 1.69 & 1.10 & 1.33 & 4.13 \\
\hline Anaxyrus punctatus & & 5 & 1 & 8.47 & 1.10 & 1.43 & 11.01 \\
\hline Incilius valliceps & & 1 & 1 & 1.69 & 1.10 & 10.30 & 13.09 \\
\hline Incilius bocourti & & 1 & 2 & 1.69 & 2.20 & 1.07 & 4.96 \\
\hline Incilius macrocristatus & & 1 & 1 & 1.69 & 1.10 & 0.83 & 3.63 \\
\hline Incilius occidentalis & & 1 & 4 & 1.69 & 4.40 & 7.47 & 13.56 \\
\hline Rhinella marina & & 5 & 8 & 8.47 & 8.79 & 9.87 & 27.13 \\
\hline \multicolumn{8}{|c|}{ FAMILIA CRAUGASTORIDAE Hedges, Duellman \& Heinicke, 2008} \\
\hline Craugastor laticeps & Pr & 1 & 1 & 1.69 & 1.10 & 3.13 & 5.93 \\
\hline Craugastor augusti & & 1 & 1 & 1.69 & 1.10 & 4.67 & 7.46 \\
\hline Craugastor glaucus & $\operatorname{Pr}$ & 1 & 1 & 1.69 & 1.10 & 3.23 & 6.03 \\
\hline
\end{tabular}




\begin{tabular}{|c|c|c|c|c|c|c|c|}
\hline & Categoría & $\begin{array}{c}\text { No. de } \\
\text { usos }\end{array}$ & $\begin{array}{c}\text { Total de } \\
\text { menciones }\end{array}$ & Lu & Fm & VuT & ICC \\
\hline Charadrahyla chaneque & $\mathrm{Pr}$ & 1 & 1 & 1.69 & 1.10 & 1.73 & 4.53 \\
\hline Dryophytes arenicolor & & 2 & 3 & 3.39 & 3.30 & 4.95 & 11.64 \\
\hline Hyla hallowellii & & 4 & 5 & 6.78 & 5.49 & 6.10 & 18.37 \\
\hline Hyla laurenti & & 2 & 1 & 3.39 & 1.10 & 11.80 & 16.29 \\
\hline Plectrohyla ixil & & 1 & 1 & 1.69 & 1.10 & 3.03 & 5.83 \\
\hline Sarcohyla bistincta & & 1 & 1 & 1.69 & 1.10 & 2.75 & 5.54 \\
\hline Smilisca manisorum & & 2 & 4 & 3.39 & 4.40 & 3.90 & 11.69 \\
\hline \multicolumn{8}{|c|}{ FAMILIA MICROHYLIDAE Günther, 1858} \\
\hline Hypopachus barberi & & 1 & 1 & 1.69 & 1.10 & 0.93 & 3.73 \\
\hline Hypopachus variolosus & & 1 & 1 & 1.69 & 1.10 & 1.70 & 4.49 \\
\hline \multicolumn{8}{|c|}{ FAMILIA RANIDAE Rafinesque, 1814} \\
\hline Lithobates berlandieri & $\operatorname{Pr}$ & 3 & 7 & 5.08 & 7.69 & 10.00 & 22.78 \\
\hline Lithobates maculatus & & 2 & 4 & 3.39 & 4.40 & 6.20 & 13.99 \\
\hline Lithobates montezumae & $\operatorname{Pr}$ & 1 & 2 & 1.69 & 2.20 & 3.63 & 7.53 \\
\hline Lithobates neovolcanicus & A & 4 & 5 & 6.78 & 5.49 & 7.00 & 19.27 \\
\hline Lithobates spectabilis & & 1 & 2 & 1.69 & 2.20 & 3.73 & 7.63 \\
\hline Lithobates zweifeli & & 3 & 3 & 5.08 & 3.30 & 5.68 & 14.06 \\
\hline \multicolumn{8}{|c|}{ FAMILIA PHYLLOMEDUSIDAE Günther, 1858} \\
\hline Agalychnis dacnicolor & & 2 & 2 & 3.39 & 2.20 & 4.52 & 10.10 \\
\hline \multicolumn{8}{|c|}{ FAMILIA PLETHODONTIDAE Gray, 1850} \\
\hline Bolitoglossa mexicana & $\operatorname{Pr}$ & 1 & 1 & 1.69 & 1.10 & 3.43 & 6.23 \\
\hline Pseudoeurycea belli & A & 1 & 1 & 1.69 & 1.10 & 4.40 & 7.19 \\
\hline \multicolumn{8}{|c|}{ FAMILIA RHINOPHRYNIDAE Günther, 1859} \\
\hline Rhinophrynus dorsalis & $\operatorname{Pr}$ & 1 & 1 & 1.69 & 1.10 & 1.57 & 4.36 \\
\hline \multicolumn{8}{|c|}{ FAMILIA SCAPHIOPODIDAE Cope, 1865} \\
\hline Spea multiplicata & & 1 & 1 & 1.69 & 1.10 & 2.30 & 5.09 \\
\hline
\end{tabular}

El IIC ofrece un valor cuantitativo que refleja las diferentes categorías de uso de la herpetofauna, lo que expresa de manera indirecta la importancia de este taxón para los pobladores y su cultura (Alcalá \& De la Riva, 2016). Por lo tanto, al identificar a los herpetozoos con mayor IIC y su relación directa con la presión de aprovechamiento extractivo, como son Crocodylus moreletii, Ctenosaura similis, Ctenosaura pectinata, Lithobates berlandieri, Lithobates neovolcanicus y Rhinella marina, se puede compreder cómo ha sido su uso histórico por las diferentes culturas (Altricher, 2006; Slotweg, 2016), su vinculación con la comunidad (indígenas o mestizas) y sus costumbres (González, 2001).

Desde tiempos prehispánicos, en Mesoamérica (Téllez, 2003) y particularmente en México (Valdéz \& Rodríguez, 2013) se ha documentado el uso de la herpetofauna por diferentes culturas (Sahagún, 1985). Por ejemplo, las serpientes es uno de los principales grupos utilizados, los cuales en la cosmovisión han adquirido un valor mágico-religioso, que les ha conferido propiedades curativas (GómezÁlvarez et al., 2007; Álvarez \& Pacheco, 2010). En contraste, otra visión de las serpientes, les han dado una connotación de animales dañinos, como animales que perjudican la salud humana y representan pérdidas económica por la muerte del ganado (Morales-Mavil \& Villa-Cañedo, 1998), por lo que son indiscriminadamente extirpadas y sus poblaciones diezmadas; aunado a lo anterior, se suman factores como el cambio de uso de suelo, contaminación, introducción de especies exóticas, cambio climático y tráfico ilegal (Aguilar \& Luría, 2016; Sánchez, 2007). Por ello se sugiere como estrategia, además de talleres de educación ambiental en escuelas rurales de educación básica y media superior, talleres participativos en las comunidades rurales en temas diversos (conocimiento y usos tradicionales, importancia ecológica, entre otros temas) que permitan acrecentar el conocimiento en la sociedad y disminuir los valores negativos resultantes de ideas erróneas sobre este grupo (Ceríaco, 2012). 
En la actualidad más de 200 especies de serpientes según la NOM-059-2010 se encuentran enlistadas en alguna categoría de riesgo de extinción, ya que sus poblaciones son demográficamente inestables; un fenómeno similar, ocurre en otros grupos taxonómicos como los lacertilia, lo cual ha implicado que la sociedad actual relacione a los anfibios y reptiles como especies nocivas o dañinas (Naranjo et al., 2010). Por lo que se ha documentado que la presión a la que está sometida la biodiversidad aumenta de forma exponencial (Heinonen et al., 2017; Verones et al., 2017). Las especies más vulnerables a las presiones antropogénicas son los anfibios, los cuales atraviesan por una situación de conservación compleja, ya que más del 50 \% de las especies se encuentran en alguna categoría de riesgo de acuerdo a la Unión Internacional para la Conservación de la Naturaleza.

Por ejemplo, del total de la herpetofauna reportada en este estudio que tienen por lo menos una mención de uso, 64 (61 \%) reptiles y 10 (31 \%) anfibios, están en la lista de especies en riesgo de la NOM-059-SEMARNAT-2010. A pesar de que existen leyes y reglamentos para evitar el saqueo y sobreexplotación de recursos naturales y que estas leyes son aplicables en materia ambiental, muy pocas veces apoyan el uso regulado de los recursos con bases biológicas y socioeconómicas, con escaso seguimiento y control en el manejo de poblaciones silvestres (Ojasti, 2000; Aldana et al., 2006). En consecuencia, el problema radica en la sociedad mexicana que carece de una cultura del cumplimiento de la ley. En este sentido, una estrategia de conservación, considerada efectiva para estos vertebrados, sería la divulgación de la importancia de estos animales en el ecosistema, su importancia en el ámbito artístico y su papel que representan en las culturas, tanto en la cosmovisión y usos (Aguilar \& Luría, 2016; González et al., 2016). Por lo tanto, la información generada en los estudios etnoherpetológicos aporta indicadores que pueden integrarse a políticas públicas de conservación y manejo.

Otorgar un valor cuantitativo por medio del IIC que tome en cuenta los usos, intensidad de uso, frecuencia de mención y valor de uso, permite que sea incorporado como un indicador de la presión de aprovechamiento; así mismo, se considera fundamental implementar estrategias de conservación basadas en el mantenimiento del hábitat y uso sustentable de las especies, mediante el esquema de las Unidades de Manejo y Conservación de la Vida Silvestre (UMA), ya sean intensivas o extensivas o Áreas Privadas para la Conservación (APC), con la finalidad de reproducirlas y de esta manera regular el tráfico ilegal y saqueo de estas especies silvestres.

Agradecimientos. A la Unidad de Manejo y Conservación de Recursos Genéticos de la Facultad de Ciencias Biológicas y Agropecuarias, región Orizaba-Córdoba, Universidad Veracruzana, por las facilidades otorgadas para el desarrollo y análisis del presente estudio.

\section{LITERATURA CITADA}

Aguilar, J., Luría, R. (2016) Los anfibios en la cultura mexicana. Ciencia, 67, 1-8.

Alves, N. R. R., van Vliet, N. (2018) Wild fauna on the Menu. Pp. 167-194. In: N. R. R. Alves (Ed.). Ethnozoology. Animals in our lives. Academic Press an imprint of Elsevier.

Albuquerque, U. P., Medeiros, P. M. (2012) Systematic reviews and meta-analysis applied to ethnobiological research. Ethnobiology and Conservation, 1, 1-8.

Alcalá, A. S., De la Riva, H. G. (2016) Uso tradicional de fauna silvestre en las serranías del occidente del Estado Aguascalientes, México. Etnobiología, 14, 20-36.

Aldana, N., Díaz, M., Feijo, A., Zuñiga, C. (2006) Valoración del uso de la fauna silvestre en el Municipio de Alcalá, Valle del Cauca. Scientia et Technica, XII, 291-297.

Álvarez, G., Pacheco, N. (2010) Uso medicinal de serpientes comercializadas en dos mercados de la Ciudad de México. Etnobiología, 8, 51-58.

Alonso-Castro, A. (2014) Use of medicinal fauna in Mexican traditional medicine. Journal of Ethnopharmacology, 152, 53-70. 
Altricher, M. (2006) Wildlife in the life of local people of the semi-arid Argentina Chaco. Biodiversity and Conservation, 15, 2719-2736.

Amador-Alcalá, S., De la Riva, H. G. (2016) Uso tradicional de fauna silvestre en las serranías del occidente del Estado Aguascalientes, México. Revista de Etnobiología, 14, 20-36.

AmphibiaWeb. (2017) https://amphibiaweb.org. University of California, Berkeley, CA, USA. Accessed May 2018.

Aranda, M., Gual-Díaz, M., Monroy-Vilchis, O., Silva, L., Velázquez, A. (1999) Aspectos etnoecológicos: aprovechamiento de la flora y fauna silvestres en el sur de la Cuenca de México. Pp. 264-283. In: A. Velázquez \& F. Romero (Eds.). Biodiversidad de la región de montaña del sur de la Cuenca de México. UNAM. Secretaría de Medio Ambiente y Recursos Naturales. México.

Ávila-Nájera D., Rosas-Rosas, O., Tarango-Arámbula, L., Martínez-Montoya, J. F., Santoyo-Brito, E. (2011) Conocimiento, uso y valor cultural de seis presas potenciales del Jaguar (Panthera onca) en San Nicolás de los Montes, San Luis Potosí. Revista Mexicana de Biodiversidad, 82, 1020-1028.

Azúa, R. V., Estrada, M. D. (2015) Cosmos, Corpus y Praxis de la Etnobiología mexicana aplicado en la educación básica. Etnobiología, 10, 52-59.

Barrasa, G. S. (2012) Conocimiento y usos tradicionales de la fauna en dos comunidades campesinas de la reserva de Biosfera de la Encrucijada, Chiapas. Etnobiología, 10, 16-28.

Balderas, C. J., Mendoza-Santos, J. F., Alvarado-Zink, A. (2014) Guía de anfibios y reptiles. Divulgación de la Ciencia y Educación Ambiental. Reserva Ecológica del Pedregal de San Ángel. Dirección General de la Divulgación de la Ciencia, UNAM.

Cabrera, A., Incháustegui, C., García, A., Toledo, V. M. (2001) Etnoecología Mazateca: Una aproximación al complejo cosmos-corpus-praxis. Etnoecológica, 6, 61-83.

Casas-Andreu, G. (2000) Mitos, leyendas y realidades de los reptiles en México. Ciencia Ergo Sum, 7, 286-291.

Casas-Andreu, G. (2008) Contribuciones al estudio de los anfibios y reptiles de México durante el siglo XVIII y la Ilustración. Ciencia Ergo Sum, 15, 101-107.

Centeno-Pérez, V. A., Arriaga-Weiss, S. L. (2010) Uso y aprovechamiento de fauna silvestre en comunidades del Parque Estatal de La Sierra, Tabasco, México. Pp. 53-77. In: M. Guerra-Roa, S. Calmé, S. Gallina-Tessaro \& E. Naranjo (Eds.). Uso y Manejo de la Fauna Silvestre en el norte de Mesoamérica. Secretaría de Educación, Instituto de Ecología, AC, ECOSUR, México.

Ceríaco, L. M. (2012) Human attitudes towards herpetofauna: The influence of folklore and negative values on the conservation of amphibians and reptiles in Portugal. Journal of Ethnobiology and Ethnomedicine, 8(1), 1-12.

Colín, H., Monroy, R. (2004) Formas de apropiación tradicional en el Corredor Biológico Chichinautzin. In: R. Monroy \& H. Colín (Eds.). Aportes Etnobiológicos Red Regional de Recursos Bióticos. Centro de Investigaciones Biológicas-Universidad Autónoma del Estado de Morelos. Cuernavaca, Morelos.

Corona, M. E. (2008) Las aves como recurso curativo en el México Antiguo y sus posibles evidencias en la arqueología. Revista de Bioarqueología ARCHAEOBIOS, 2, 11-18.

Cortés-Gregorio, I., Pascual-Ramos, E., Medina-Torres, S. M., Sandoval-Forero, E. A. (2013) Etnozoología del pueblo Mayo-Yoreme en el norte de Sinaloa: uso de vertebrados silvestres. Agricultura Sociedad y Desarrollo, 10, 335-358.

Cossio, B. A. (2007) Conocimiento y comparación del uso de la fauna silvestre en dos comunidades ejidales del municipio de Hueytamalco, Puebla, México. Tesis de Maestría. Instituto de Ecología A. C., Xalapa, Veracruz.

Cupul-Magaña, F. G. (2003) Cocodrilo: medicina para el alma y el cuerpo. Revista Biomédica, 14, 45-48. 
Cháble, S. H., Delfín, G. H. (2010) Uso tradicional de la fauna silvestre. Pp. 377-381. In: R. Durán \& M. Méndez (Eds.). Biodiversidad y Desarrollo Humano en Yucatán. CICY, PPD-FMAM, CONABIO, SEDUMA. México.

Enríquez-Vázquez, P., Mariaca-Méndez, M., Retana-Guiascón, O., Naranjo-Piñera, J. (2006) Uso medicinal de la fauna silvestre en los altos de Chiapas, México. Interciencia, 31, 491-499.

Flores-Villela, O., García-Vázquez, U. O. (2013) Biodiversidad de reptiles en México. Revista Mexicana de Biodiversidad, 84, 467-475.

Frost, D. R. (2018) Amphibian Species of the World: an Online Reference. Version 6.0 (May 16, 2018). Electronic Database accessible at http://research.amnh.org/herpetology/amphibia/index.html. American Museum of Natural History, New York, USA.

García, F. A., Monroy, R. (2010) Conocimiento, uso y manejo de fauna cinegética de la Reserva Estatal Sierra de Montenegro, Morelos. In: E. B. O. Villareal, H. J. Hernández, R. J. Camacho \& G. F. Franco (Coord.). Conservación y manejo de fauna cinegética de México II. Benemérita Universidad Autónoma de Puebla. Fundación Produce Puebla A. C. Mazamiztli, A. C. México.

González, T. Y. (2001) Lo animal en la cosmovisión mexica o mesoamericana. Pp. 23-51. In: T. Y. González (Ed.). Animales y plantas en la cosmovisión mesoamericana. Plaza y Valdés, Instituto Nacional de Antropología e Historia y Sociedad Mexicana para el Estudio de las Religiones. México, D. F.

Gómez-Álvarez, G., Pacheco, N. (2010) Uso medicinal de serpientes comercializadas en dos mercados de la ciudad de México. Etnobiologia, 8(1), 51-58.

Gómez-Álvarez, G., Reyes-Gómez, S. R., Teutli-Solano, C., Valadez-Azúa, R. (2007) La medicina tradicional prehispánica, vertebrados terrestres y productos medicinales de tres mercados del Valle de México. Etnobiología, 5, 86-98.

Gómez-Landa, N. (2015) Uso de fauna silvestre en el ejido Pinoltepec, Municipio de Emiliano Zapata, Veracruz. Tesis de Licenciatura. Universidad Veracruzana, Xalapa, Veracruz.

González-Bocanegra, K., Romero-Berny, E., Escobar-Ocampo, M. C., García-Del Valle, Y. (2011) Aprovechamiento de fauna silvestre por comunidades rurales en los humedales de Catazajá - La Libertad, Chiapas, México. Ra Ximhai, 7, 219-230.

González, J. A., Amich, F., Postigo-Mota, S., Vallejo, J. R. (2016) The use of wild vertebrates in contemporary Spanish ethnoveterinary medicine. Journal of Ethnopharmacology, 191, 135-151.

Guerrero, S., Retana, O. (2012) Nota científica: uso medicinal de la fauna silvestre por indígenas tlahuicas en Ocuilan, México. Etnobiología, 10, 28-33.

Heinonen, T., Pukkala, T., Mehtätalo, L., Asikainen, A., Kangas, J., Peltola, H. (2017) Scenario analyses for the effects of harvesting intensity on development of forest resources, timber supply, carbon balance and biodiversity of Finnish forestry. Forest Policy and Economics, 80, 80-98.

Hernández-López, A., López-Alamilla, E., Rodríguez-Ramírez, A., Aquino-Brabata, V. (2013) Diagnóstico del uso de la fauna silvestre, en el área de Protección de Flora y Fauna "Cañón de Usumacinta”, Tenosique, Tabasco. Ra Ximhai, 9, 1-14.

Jacobo-Salcedo, M. R., Alonso-Castro, A. J., Zarate-Martínez, A. (2010) Folk medicinal use of fauna in Mapimí, Durango, México. Journal of Ethnopharmacology, 122, 902-906.

Juárez-Mondragón, A., González-Rebeles, C., Castillo, A., García-Frapolli, E., Ordoñez-Díaz, M. J. (2015) La vida silvestre manejada como recursos de uso común: estudio de caso en México. Tropical and Subtropical Agroecosystems, 18, 313-331.

León-Martínez, P. (2006) Aprovechamiento de fauna silvestre en una comunidad aledaña a la Reserva de la Biosfera Los Petenes, Campeche. Tesis de Maestría. Centro de Investigaciones y de Estudios Avanzados del Instituto Politécnico Nacional, Mérida, México.

León, P., Montiel, S. (2009) Wild meat use and traditional hunting practices in a rural Mayan community of the Yucatan Peninsula, Mexico. Human Ecology, 36, 249-257.

Leyte-Manrique, A., Gutiérrez-Álvarez, N., Hernández-Navarro, E. M. (2016) Percepción cultural de la herpetofauna en tres comunidades rurales del municipio de Irapuato, Guanajuato, México. Etnobiología, 14, 73-84. 
Lira-Torres, I., Ojera-Ramírez, D., Peláez Acero, A., Briones-Salas, M., Gómez de Anda, F., OjedaRamírez, D., Peláez-Acero, A. (2014) Usos y aprovechamiento de la fauna silvestre en la selva Zoque, México. Acta Zoológica Mexicana (nueva serie), 30, 74-90.

Macip-Ríos, R., Casas-Andreu, G. (2008) Los cafetales en México y su importancia para la conservación de los anfibios y reptiles. Acta Zoológica Mexicana (nueva serie), 24, 143-159.

March, I. J., Naranjo E. J., Rodiles R., Navarrete D. A., Alba, M. P., Hernández, P. J. (1996) Diagnóstico para la conservación y manejo de la fauna silvestre en la selva Lacandona, Chiapas. Informe final para la Secretaría de Medio Ambiente, Recursos Naturales y Pesca (SEMARNAP). El Colegio de la Frontera Sur, San Cristóbal de Las Casas, Chiapas, México.

Martínez-Ibarra, J., Arellano-Montoya, R. (2010) Uso comunitario de los recursos naturales en el ejido Lagunillas, Jalisco, México. Ambiente y Desarrollo, XIV, 95-109.

Méndez, F. (2005) Estudio preliminar de aprovechamiento de la flora y fauna silvestre en dos comunidades del norte de Campeche. Tesis de Licenciatura. Universidad Veracruzana. Xalapa, México.

Méndez-Cabrera, F., Montiel, S. (2007) Diagnóstico preliminar de la fauna y flora silvestre utilizada por la población maya de dos comunidades costeras de Campeche, México. Universidad y Ciencia, 23, 127-139.

Millán-Rojas, L., Arreaga-Reyes, T. T., Moctezuma-Pérez, S., Velasco-Orozco, J. J., ArzateSalvador, J. C. (2016) Conocimiento ecológico tradicional de la biodiversidad de bosques en una comunidad de Matlantzinca, México. Ambiente y Desarrollo, XX, 111-123.

Monroy, R., García, A. (2013) La fauna silvestre con valor de uso en los huertos frutícolas tradicionales de la comunidad Indígena de Xoxocotla, Morelos, México. Etnobiología, 11, 44-52.

Monroy-Vilchis, O., Cabrera, L., Suárez, P., Zarco-González, M. M., Rodríguez-Soto, C., Urios, V. (2008) Uso tradicional de vertebrados silvestres en la Sierra Nanchititla, México. Interciencia, 33, 308-317.

Montiel-Ortega, S., Arias-Reyes, L., Dicknson, F. (1999) La cacería tradicional en el norte de Yucatán: una práctica comunitaria. Revista de Geografía Agrícola, 29, 43-52.

Morales-Maví, J., Suárez-Castillo, S. (2010) Conocimiento y uso de tortugas de agua dulce por pescadores y lancheros de Sontecomapan, Veracruz, México. Pp. 193-261. In: M. Guerra-Roa, S. Calmé, S. Gallina-Tessaro \& E. Naranjo (Eds.). Uso y Manejo de la Fauna Silvestre en el norte de Mesoamérica. Secretaria de Educación, Instituto de Ecología, AC, ECOSUR, México.

Morales-Mavil, J. E., Villa-Cañedo, T. (1998) Notas sobre el uso de la fauna silvestre en Catemaco, Veracruz, México. Acta Zoológica Mexicana (nueva serie), 73, 127-143.

Naranjo, E. (2013) Uso de la fauna silvestre. Pp. 271-280. In: A. Cruz-Angón, E. D. Melgarejo, Camacho- F. Rico \& K. C. Nájera-Conchero (Eds.). La Biodiversidad de Chiapas: Estudio de Estado. Comisión Nacional para el Conocimiento y Uso de la Biodiversidad y Gobierno del Estado de Chiapas. D. F. México.

Naranjo, E., Cuarón, A. D. (2010) Usos de la fauna silvestre. Pp. 271-283. In: G. Ceballos, L. Martínez, A. García, E. Espinoza, J. Bezaury \& R. Dirzo (Eds.). Diversidad, amenazas y áreas prioritarias para la conservación de las selvas secas del Pacífico de México. Fondo de Cultura Económica y CONABIO. México, D. F.

Naranjo, E., Guerra, M., Bodmer, R., Bolaños, J. (2004) Subsistence hunting by three ethnic groups of the Lacandon Forest, Mexico. Journal of Ethnobiology, 24, 233-253.

Naranjo, E., Guerra-Roa, M., Gallina-Tessaro, S., Calmé, S. (2010) Uso de fauna silvestre en el norte de Mesoamérica: aspectos generales. Pp. 19-24. In: M. Guerra-Roa, S. Calmé, S. Gallina-Tessaro \& E. Naranjo (Eds.). Uso y manejo de Fauna Silvestre en el norte de Mesoamérica. Instituto de Ecología, A.C., ECOSUR. México.

Navarijo-Ornelas, M. L. (2004) Presencia e importancia de los animales en la medicina tradicional de los grupos otopames. Estudios de Cultura Otopame, 4, 197-214. 
Nucadami, L. (2006) Uso de los vertebrados terrestres en dos comunidades indígenas de la selva lacandona, Chiapas, México. Tesis de Licenciatura. Universidad de Ciencias y Artes de Chiapas. Tuxtla Gutiérrez, Chiapas.

Ojasti, J. (2000) Manejo de fauna silvestre neotropical. SIMAB Series $\mathrm{N}^{\circ}$ 5. Smithsonian Institution / MAB Program. Washington, D. C.

Parra-Olea, G., Flores-Villela, O., Mendoza-Almeralla, C. (2014) Biodiversidad de anfibios en México. Revista Mexicana de Biodiversidad, 85, S460-S466.

Pascual-Ramos, E., Medina-Torres, S. M., Sandoval-Forero, E., Lara-Ponce, E., Piña-Ruíz, H., Martínez-Ruíz, R., Rojo-Martínez, G. (2014) Uso de reptiles entre Yoremes y Yoris en el municipio de el fuerte, Sinaloa. Ra Ximhai, 10, 185-208

Penguilly, M. M., Moreno, F. Á., Goyenechea, M. I., Espinoza, P. G. (2010) Percepción acerca de las lagartijas consideradas nocivas por algunos Otomíes, Nahuas, Tepehuas y Mestizos en el estado de Hidalgo, México. Pp. 99-105. In: A. Moreno, R. Valadéz, M. T. Pulido, R. Mariaca, P. Mejía \& S. V. Gutiérrez (Eds.). Etnobiología y sistemas biocognitivos tradicionales: paradigmas en la conservación biológica y el fortalecimiento cultural. Universidad Autónoma del Estado de Hidalgo, Asociación Etnobiológica Mexicana y Sociedad Latinoamericana de Etnobiología. México.

Ramírez-Mella, M., Candelaria-Martínez, B., Dorantes-Jiménez, J., Tarango-Arámbula, L. A., Flota-Bañuelos, C. (2016) Uso y aprovechamiento de fauna silvestre en zonas rurales de Campeche, México. Agroproductividad, 9, 3-9.

Reyna-Rojas, M. A., García, F. A., Neri, C. E. E., Alargón, C. A., Monroy, M. R. (2015) Conocimiento etnoherpetológico de dos comunidades aledañas a la Reserva Estatal Sierra de Montenegro, Morelos, México. Etnobiología, 13, 37-48.

Rodríguez-Soto, C., Velazquez, A., Monroy-Vilchis, O., Lemes, P., Loyola, R. (2017) Joint ecological, geographical and cultural approach to identify territories of opportunity for large vertebrates conservation in Mexico. Biodiversity and Conservation, 26, 1899-1918.

Sahagún, B. (1985) Historia general de las cosas de la Nueva España. Ed. Porrúa, México.

Sánchez, O. (2007) Método de evaluación del riesgo de extinción de las especies silvestres en México (MER). Instituto Nacional de Ecología. México.

Sánchez, E. (2006) Conocimiento tradicional Mazahua de la herpetofauna, un estudio etnozoológico de la Reserva de la Biósfera Mariposa Monarca, México. Estudios Sociales, 15, 43-46.

Santos-Fita, A., Naranjo, E., Rangel-Salazar, J. L. (2012) Wildlife uses and hunting patterns in rural communities of the Yucatan Peninsula, Mexico. Journal of Ethnobiology and Ethnomedicine, 8, $3-17$.

SEMARNAT. (2010) Norma Oficial Mexicana NOM-059-SEMARNAT-2010. Protección ambiental, especies nativas de flora y fauna silvestres de México, categorías de riesgo y especificaciones para su inclusión, exclusión o cambio, y lista de especies en riesgo. Diario Oficial de la Federación. D. F. México.

Serrano-González, R., Guerrero-Martínez, F., Serrano-Velazquez, R. (2011) Animales medicinales y agoreros entre tzotziles y tojolabales. Estudios Mesoamericanos, 11, 29-42.

Slootweg, J. C. (2016) ¿Conflictos socio-culturales o resignificación de prácticas simbólicas? Revisión de estudios etnográficos de interculturalidad religiosa: América Latina y El Caribe. Interciencia, 41, 362-368.

Tejeda-Cruz, C., Naranjo-Piñera, E. J., Medina-Sanson, L. M., Guevara-Hernández, F. (2014) Cacería de subsistencia en comunidades rurales de la selva Lacandona, Chiapas, México. Quehacer Científico de Chiapas, 9, 59-73.

Téllez, E. (2003) Acercamiento al estudio de los animales en las culturas prístinas de Mesoámerica. Imagen Veterinaria, 3, 21-29.

Toledo, V. M., Barrera, B. N. (2008) La memoria biocultural. La importancia ecológica de las sabidurías tradicionales. Icaria editorial, Barcelona, España. 
Turner, N. (1988) The importance of a rose. Evaluating the cultural significance of plants in Thompson and Lilloet Interior Salish. American Anthropologist, New Series, 90, 272-290.

Turtle Taxonomy Working Group (Rhodin, A. G. J., Iverson, J. B., Bour, R., Fritz, U., Georges, A., Shaffer, H. B., van Dijk, P. P.). (2017) Turtles of the World: Annotated Checklist and Atlas of Taxonomy, Synonymy, Distribution, and Conservation Status (8th Ed.). In: A. G. J. Rhodin, J. B. Iverson, P. P. van Dijk, R. A. Saumure, K. A. Buhlmann, P. C. H. Pritchard \& R. A. Mittermeier (Eds.). Conservation Biology of Freshwater Turtles and Tortoises: A Compilation Project of the IUCN/SSC Tortoise and Freshwater Turtle Specialist Group. Chelonian Research Monographs. 7, 1-292. doi: 10.3854/crm.7.checklist.atlas.v8.2017.

Uetz, P., Freed, P., Hošek, J. (2018) The Reptile Database, http://www.reptile-database.org. Accessed May, 2018.

Uriarte-Garzón, P. (2012) El conocimiento etnoherpetológico dentro de las comunidades del Área Natural Protegida Cerro de Arandas, Irapuato. Pp. 241-243. In: Comisión Nacional para el Conocimiento y Uso de la Biodiversidad (Ed.) La biodiversidad en Guanajuato: Estudio de Estado. Comisión Nacional para el Conocimiento y Uso de la Biodiversidad e Instituto de Ecología del Estado de Guanajuato. México.

Valdéz, R., Rodríguez, B. (2013) Uso de la fauna, estudios arqueozoológicos y tendencias alimentarias en culturas Prehispánicas del centro de México. Anales de Antropología, 48, 139-166.

Vásquez-Dávila, M. A. (1999) La etnobiología en México. Reflexiones y experiencias. Instituto Tecnológico Agropecuario de Oaxaca. Asociación Etnobiológica Mexicana, SEP, CONACYT, Oaxaca, México.

Vázquez-Días, J., Quintero-Días, G. (2012) Etnozoología. Pp. 227-230. In: Comisión Nacional para el Conocimiento y Uso de la Biodiversidad (Ed.). La Biodiversidad en Aguascalientes: Estudio de Estado. Comisión Nacional para el Conocimiento y Uso de la Biodiversidad, Instituto del Medio Ambiente del Estado de Aguascalientes (IMAE), Universidad Autónoma de Aguascalientes (UAA). Aguascalientes, México.

Verones, F., Moran, D., Stadler, K., Kanemoto, K., Wood, R. (2017) Resource footprints and their ecosystem consequences. Scientific Reports, 7, 40743. 1-12. 